PHYSICS ESSAYS ( Volume 25, Issue 2 (June 2012) ) P172 176

URL: http://physicsessays.org/toc/phes/25/2

\title{
Entropy: A concept that is not a physical quantity
}

\author{
Shufeng-zhang \\ College of Physics, Central South University, 932 Lushan south Road, Changsha 410083, China \\ E-mail: uhsgnahz@,126.com
}

\begin{abstract}
This study has demonstrated that entropy is not a physical quantity, that is, the physical quantity called entropy does not exist. If the efficiency of heat engine is defined as $\eta=W / W_{1}$, and the reversible cycle is considered to be the Stirling cycle, then, given $\oint \mathrm{dQ} / \mathrm{T}=0$, we can prove $\oint \mathrm{dW} / \mathrm{T}=0$ and $\oint \mathrm{d} / \mathrm{T}=0$. If $\oint \mathrm{dQ} / \mathrm{T}=0, \oint \mathrm{dW} / \mathrm{T}=0$ and $\oint \mathrm{dE} / \mathrm{T}=0$ are thought to define new system state variables, such definitions would be absurd. The fundamental error of entropy is that in any reversible process, the polytropic process function $\mathrm{Q}$ is not a single-valued function of $\mathrm{T}$, in thermodynamics, the P-V diagram should be P-V-T diagram, and the key step of $\Sigma[(\Delta \mathrm{Q}) / \mathrm{T})]$ to $\int \mathrm{dQ} / \mathrm{T}$ doesn't hold. Similarly, $\oint \mathrm{dQ} / \mathrm{T}=0, \oint \mathrm{dW} / \mathrm{T}=0$ and $\oint \mathrm{dE} / \mathrm{T}=0$ do not hold, either. Since the absolute entropy of Boltzmann is used to explain Clausius entropy and the unit $(\mathrm{J} / \mathrm{K})$ of the former is transformed from the latter, the non-existence of Clausius entropy simultaneously denies Boltzmann entropy.
\end{abstract}

Résumé : Prouver que ‘entropie'n'est pas de la grandeur physique, c'est-à-dire, il n'existe pas le soi-disant 'entropie' cette grandeur physique. Lorsque nous définissons le rendement thermique comme $\eta=W / W_{1}$, alors que le cycle réversible devient le cycle de Stirling, si $\oint \mathrm{dQ} / \mathrm{T}=0$, nous pouvons prouver que $\oint \mathrm{dW} / \mathrm{T}$ $=0, \oint \mathrm{dE} / \mathrm{T}=0$. Si nous pensons que $\oint \mathrm{dQ} / \mathrm{T}=0, \oint \mathrm{dW} / \mathrm{T}=0$ et $\oint \mathrm{dE} / \mathrm{T}=0$ définissent la nouvelle quantité d'état du système, alors ceci montre que telle définition doit être ridicule. L'erreur basique réside en ce que la function du processus polytropique $\mathrm{Q}$ dans n'import quel process réversible n'est pas une function uniforme T, donc l'étape clé $\left.\sum[(\Delta \mathrm{Q}) / \mathrm{T})\right]$ est écrit comme $\int \mathrm{dQ} / \mathrm{T}$ est false. Puisque l'entropie absolue de Boltzmann est pour expliquer l'entropie de Clausius, l'unité de l'entropie de Boltzmann(J/K) est aussi migrée de l'entropie de Clausius,la nonexistance de l'entropie de Clausius nie en même temps l'entropie de Boltzmann.

Key words: entropy; thermodynamics; statistical physics 


\section{Introduction}

What is entropy? This question has been debated for over 100 years.

Historically, Clausius proposed the existence of new system state variable entropy (S) in 1865 based on the equation $\oint d Q / T=0$ obtained from the reversible cycle of thermodynamic system. He considered the entropy difference between any two equilibrium states of a thermodynamic system as: ${ }^{1,2,3,4,5}$

$$
\Delta S=S_{2}-S_{1}=\int_{1}^{2} d Q / T
$$

Only such a difference can be calculated in thermodynamics. Correspondingly, Clausius proposed the well-known law of entropy increase.

Subsequently, Boltzmann proposed the formula $S=k \ln \Omega^{1,2,3}$ for absolute entropy in 1872 , where $\mathrm{k}$ is the Boltzmann constant and $\Omega$ the thermodynamic probability. In addition, Boltzmann suggested entropy as the degree of disorder in a system or the measurement mark of order. This is considered to be the best interpretation of entropy and has been in use till today.

The above conclusions, which can be found in many textbooks of thermodynamics and statistical physics, are presently widely accepted and learned. Despite the uncertainty in what entropy really is, entropy has commonly been used as an important physical quantity.

However, there are still many unresolved problems and contradictions difficult to justify in the above conclusions. This indicates entropy is problematic.

\section{Entropy is not a physical quantity}

\section{A. The origin of entropy}

To illustrate that entropy is not a physical quantity, we will briefly review its origin.

First, the efficiency of heat engine is defined as $\eta=W / Q_{1},{ }^{1,2,3,4,5}$ that is, taking the ratio of $\mathrm{W}$ to $Q_{1}$ as the efficiency of heat engine, where $\mathrm{W}$ is the net work done by system to the outside world and $Q_{1}$ the heat absorbed by the system from the outside world in a heat engine cycle. Regarding the Carnot cycle, there is $\eta=W / Q_{1}=1-Q_{2} / Q_{1}$, where $\eta$ is related to the constant temperature of two heat sources, 
rather than the working substance of the system. Accordingly, the thermodynamic temperature scale $\theta$ is defined as $\theta_{2} / \theta_{1}=Q_{2} / Q_{1}$. When an ideal gas is the working substance of the system, we can prove that:

$$
Q_{2} / Q_{1}=T_{2} / T_{1}, \text { that is, } \theta_{2} / \theta_{1}=T_{2} / T_{1}
$$

The symbol $\mathrm{T}$ is used to represent the thermodynamic temperature scale, that is, $Q_{2} / Q_{1}=T_{2} / T_{1} \Rightarrow Q_{1} / T_{1}+Q_{2} / T_{2}=0$, where $Q_{2}$ is the heat released (negative value). Then, the arbitrary processes of reversible cycles are approached and replaced by an infinite number of Carnot cycles, resulting in the equation $\oint d Q / T=0 .{ }^{1,2,3,4,5}$ So far as it goes, $d Q / T$ is considered as a complete differential and the new system status quantity entropy is determined by $\oint d Q / T=0$.

\section{B. Entropy is not a physical quantity}

Entropy comes from $\oint d Q / T=0$. Thus, to prove entropy is not a physical quantity, we only need to prove that $\oint d Q / T=0$ cannot define a physical quantity or it is untenable in itself.

As we known, $\oint d Q / T=0$ comes from the equation $Q_{2} / Q_{1}=T_{2} / T_{1}$ in Carnot cycle, which has been used to define the thermodynamic temperature scale. The basis of its existence lies in the combination of Carnot cycle and definition of the efficiency of heat engine. It should be noticed that the equation for the efficiency of heat engine is a definition, and the Carnot cycle is only different from other reversible cycles by the form. Therefore, Carnot cycle should not occupy a status higher than other forms of cycles, and its role in defining the thermodynamic temperature scale is not unique.

In this section we will prove that $\oint d Q / T=0$ cannot define a physical quantity and it is untenable in itself.

First, the efficiency of heat engine needs to be redefined.

As the efficiency of heat engine is meaningful only to the observer, how we define it is not related to objective processes of the heat engine system. Therefore, the efficiency of heat engine can reasonably be defined via other pathways. Here, we redefine the efficiency of heat engine as the ratio of the net work to work done by the heat engine system to the external world in a cycle: 


$$
\eta=\frac{W}{W_{1}}
$$

That is, the work $W_{1}$ done by the system to the external world in a cycle will replace the heat $Q_{1}$ absorbed by the system from the external world in the original definition $\eta=W / Q_{1}$. As the work $W_{1}$ done by the system to the external world in a cycle cannot entirely be transformed into net work $W$, and likewise, the heat absorbed by the system from the external world in a cycle cannot entirely be used to do net work to the outside - Kelvin's statement of the second law, these two definitions apparently have the same meaning. Here the second law is expressed in another form: no such an engine can transform all its work to net work to the external world in a cycle. Apparently, this is equivalent to the Kelvin's expression.

For example, a heat engine uses a certain amount of working substance to do work $W_{1}$ to the external world in a cycle, and the external world does work $W_{2}$ to the system for recovery. Then,

$$
W=W_{1}-W_{2}
$$

From ( 1 ) we find:

$$
\eta=\frac{W}{W_{1}}=1-\frac{W_{2}}{W_{1}}
$$

If the Stirling reversible cycle (Figure 1) is taken as the unit cycle, it will play the role of Carnot cycle in the deduction of $\oint d Q / T=0$.

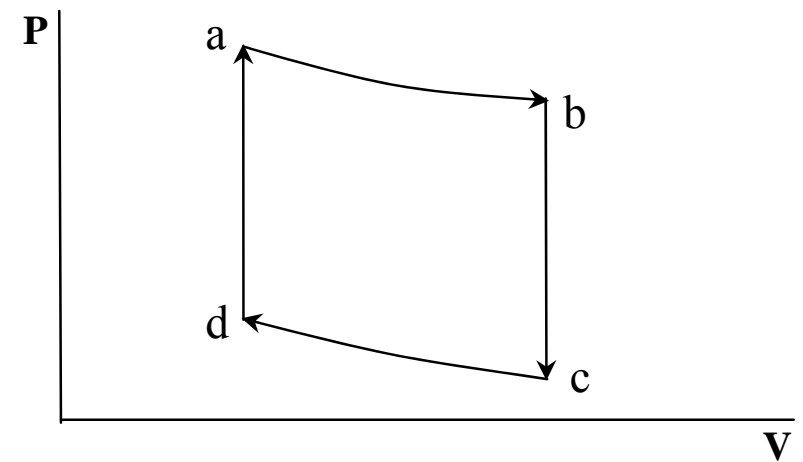

Fig. 1 . TV cycle (Stirling cycle). abeda consist of two reversible isochoric processes (bc and da), and two reversible isothermal processes ( $\mathrm{ab}$ and $\mathrm{cd})$. The heat source, which exchanges energy power with the system in an isothermal process, is considered as the work source for a better understanding of the followings. This cycle is referred to as TV cycle for short, and the heat engine doing TV cycle (Stirling cycle) is the TV engine. 
In this section we will prove that all reversible engines (TV engines) only working between two work sources with constant temperature share the same efficiency, and the efficiency of irreversible engines is lower than that of reversible engines.

Taking any two reversible engines $\mathrm{E}$ and $\mathrm{E}^{\prime}$ for example, both engines work between two work sources with constant temperature $\theta_{1}$ and $\theta_{2}$. They are certainly TV engines and have arbitrary working substance. Given that $\theta_{1}$ and $\theta_{2}$ represent the high- and low-temperature work sources, respectively, we get $\theta_{1}>\theta_{2}$. Here, $\theta$ can take any temperature scales. If we let $\mathrm{E}$ and $\mathrm{E}^{\prime}$ do the same net work ( $\Delta \mathrm{W}_{1}$ and $\Delta \mathrm{W}_{2}$, respectively) to the external world in a cycle, then we will find $\Delta \mathrm{W}_{1}=\Delta \mathrm{W}_{2}=\mathrm{W}$ (similar to Carnot cycle). Using $\mathrm{W}_{1}$ and $\mathrm{W}_{1}{ }^{\prime}$ to represent the work done by $\mathrm{E}$ and $\mathrm{E}$ ' in a cycle, $\mathrm{W}_{2}$ and $\mathrm{W}_{2}{ }^{\prime}$ to represent the work done by the external world to $E$ and $E$ ', and $\eta$ and $\eta$ ' to represent the efficiency of $E$ and $E$ ', we will first prove $\eta^{\prime} \leq \eta$ by contradiction:

Assuming $\quad \eta^{\prime}>\eta$

As $\mathrm{E}$ and $\mathrm{E}^{\prime}$ are both reversible, we let $\mathrm{E}$ do reverse movement. Then, $\mathrm{W}_{2}$ is the work done by $\mathrm{E}$ to the external world, $\mathrm{W}_{1}$ the work done by the external world to $\mathrm{E}$, and $\mathrm{W}$ the net work done by the external world to $\mathrm{E}$. We have $\mathrm{W}=\mathrm{W}_{1}-\mathrm{W}_{2}$. W is provided by $\mathrm{E}^{\prime}$, and the heat absorbed by $\mathrm{E}^{\prime}$ in the cycle $\Delta \mathrm{Q}=\mathrm{W}$ $\left(=\Delta \mathrm{W}_{1}=\Delta \mathrm{W}_{2}\right)$ provided by $\mathrm{E}$. Thus,

$$
\frac{W}{W_{1}{ }^{\prime}}>\frac{W}{W_{1}} \Rightarrow W_{1}>W_{1}{ }^{\prime}
$$

Also, because

$$
\mathrm{W}_{2}=\mathrm{W}_{1}-\mathrm{W} \quad \text { and } \quad \mathrm{W}_{2}{ }^{\prime}=\mathrm{W}_{1^{\prime}-\mathrm{W}}
$$

Thus,

$$
\mathrm{W}_{2}>\mathrm{W}_{2}{ }^{\prime}
$$

If we incorporate $\mathrm{E}^{\prime}$ and reversely-moving $\mathrm{E}$ into one heat engine, the only result of the system recovery after a combined cycle is that the system absorbs work $\left(\Delta \mathrm{W}=\mathrm{W}_{2}-\mathrm{W}_{2}{ }^{\prime}\right)$ from the low-temperature work source (heat source $\left.\theta_{2}\right)$ and automatically does work $\left(\Delta \mathrm{W}=\mathrm{W}_{1}-\mathrm{W}_{1^{\prime}}=\mathrm{W}_{2}-\mathrm{W}_{2}{ }^{\prime}\right)$ to the high-temperature work source (heat source $\theta_{1}$ ). That is, there is equal heat $\Delta \mathrm{W}=\mathrm{W}_{1}-\mathrm{W}_{1^{\prime}}=\mathrm{W}_{2}-$ $\mathrm{W}_{2}{ }^{\prime}$ automatically sent from the low- (heat source $\theta_{2}$ ) to the high-temperature work source (heat source $\theta_{1}$ ). This is directly in contradiction with Clausius' expression of the second law, indicating $\eta^{\prime}>\eta$ doesn't hold. Likewise, we can prove $\eta>\eta$ is untenable based on the assumption regarding reversely moved E'. So, we should have: 


$$
\eta=\eta^{\prime}
$$

If $\mathrm{E}^{\prime}$ is an irreversible engine, i.e., non-TV engine, then $\mathrm{E}^{\prime}$ cannot move reversely. There should be:

$$
\eta^{\prime} \leqslant \eta
$$

Given that $E^{\prime}$ is an irreversible engine, and that a combined cycle of $E^{\prime}$ and the reversible engine $E$ recovers the system and the external world, the equal mark in $\eta^{\prime} \leq \eta$ doesn't hold upon the premise of irreversible engine $E^{\prime}$. This is because, if we have $\eta=\eta^{\prime}$, a combined cycle of reversely-moving $E$ and forwardly-moving $E^{\prime}$ will obviously completely recover the system and the external world. Then, E' can only be a reversible engine, in contradiction to the fact that $\mathrm{E}^{\prime}$ is an irreversible engine. Therefore, if $\mathrm{E}^{\prime}$ is an irreversible engine, we should have:

$$
\eta^{\prime}<\eta
$$

In this way, according to the definition (1), we have proved that all reversible engines (TV engines) that only work between two work sources with constant temperature share the same efficiency. The efficiency of irreversible engine is lower than that of reversible engine, showing no relationship with the working substance.

As the efficiency of TV engine is not related to working substance, the thermodynamic temperature scale (absolute thermometric scale) can be defined as:

$$
\frac{\theta_{1}}{\theta_{2}}=\frac{W_{1}}{W_{2}}
$$

That is, the ratio of two thermodynamic temperatures is the ratio of the work $\mathrm{W}_{1}$ and $\mathrm{W}_{2}$ exchanged between the TV engine working between two work sources with constant temperature (heat sources) and the work sources.

When the working substance is an ideal gas and the system does TV cycle, we have:

$$
\eta=1-\frac{W_{2}}{W_{1}}=1-\frac{\int_{V_{2}}^{V_{1}} P d V}{\int_{V_{2}}^{V_{1}} P^{\prime} d V^{\prime}}=1-\frac{v R T_{2} \ln \frac{V_{2}}{V_{1}}}{v R T_{1} \ln \frac{V_{2}}{V_{1}}}=1-\frac{T_{2}}{T_{1}}
$$


By comparing (6) and (7), we obtain $\theta_{2} / \theta_{1}=T_{2} / T_{1}$ for the ideal gas, that is, the thermodynamic temperature scale defined by (6) is equivalent to the thermodynamic temperature scale defined by $\theta_{2} / \theta_{1}=Q_{2} / Q_{1}$. Still, we use symbol $\mathrm{T}$ to represent the thermodynamic temperature scale:

$$
\frac{T_{1}}{T_{2}}=\frac{W_{1}}{W_{2}}
$$

It can be deduced from (8) that

$$
\frac{W_{1}}{T_{1}}+\frac{W_{2}}{T_{2}}=0
$$

where $\mathrm{W}_{2}$ is the work that the external world has done to the system (negative value). This indicates that when any system does TV cycles, the sum of the work (positive or negative value) exchanged between the system and each work source (heat source) and the thermodynamic temperature ratio of the work source is zero.

Following the exactly same process of deduction of $\oint d Q / T=0$, a serial element TV cycles are employed to split and replace any reversible cycles in the system (Figure 2).

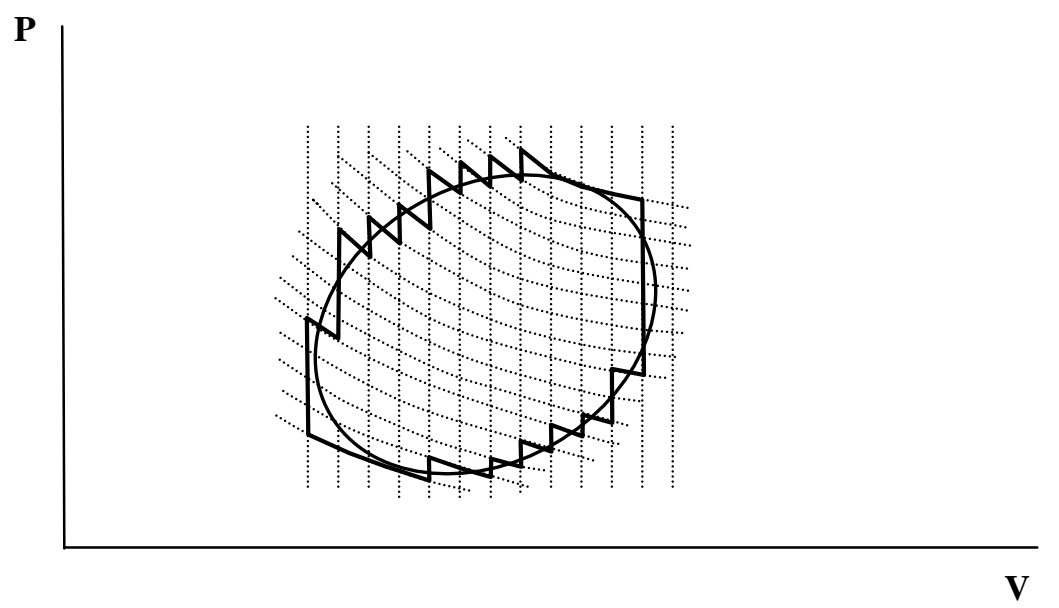

Fig. 2. Diagram of the process of any reversible cycles split and replaced by a series of element TV cycles

In two isochoric processes of TV cycle, there is always $\Delta \mathrm{W}=0$ (i.e., $\mathrm{dW}=0$ ). Thus, when there are infinite element processes, or the system exchanges work (equivalent to heat) with infinite work sources (heat sources), we get:

$$
\oint \frac{d W}{T}=0
$$


Again, this is not related to the working substance of the system.

With respect to the irreversible cycle, we obviously can deduce the conclusion $\oint \frac{d W}{T}<0$.

So far, we have obtained the conclusion $\oint d \mathrm{~W} / T=0$, which is paratactic with $\oint d Q / T=0$. Then, according to the first law $d E=d Q+d W$, we can obtain the following conclusion for reversible cycles in any thermodynamic system:

$$
\oint \frac{d E}{T}=\oint \frac{d Q}{T}+\oint \frac{d W}{T}=0
$$

Over the last century, $\oint d Q / T=0$ has been thought to define a system state variable, i.e., entropy.

Then, we get:

$$
\oint \frac{d W}{T}=0 \text { and } \oint \frac{d E}{T}=0
$$

This certainly defines a new system state variable. In addition, taking the reversible adiabatic process for example, when the system approaches a different equilibrium state 2 from equilibrium state 1 , we should have:

$$
\begin{aligned}
& \int_{1}^{2} \frac{d Q}{T} \neq \int_{1}^{2} \frac{d W}{T} \\
& \int_{1}^{2} \frac{d Q}{T} \neq \int_{1}^{2} \frac{d E}{T}
\end{aligned}
$$

During the reversible isochoric process of the system, the following equation exists for different equilibrium states 3 and 4 ,

$$
\int_{3}^{4} \frac{d W}{T} \neq \int_{3}^{4} \frac{d E}{T}
$$

As shown in (11) (13), if we let $\oint \frac{d Q}{T}=0, \oint \frac{d W}{T}=0$ and $\oint \frac{d E}{T}=0$ define a new system state variable, they should be different from each other, despite the same dimension $(\mathrm{J} / \mathrm{K})$. Therefore, there is a state variable in the system with different values; or there are three different state variables in the 
system with the same unit. Although different state variables with the same unit are likely to occur in a system, it is absurd to define three variables due to the uncertainty in the only one S.

Thus, $\oint \frac{d Q}{T}=0$ cannot define a physical quantity.

Similarly, $\oint \frac{d W}{T}=0$ and $\oint \frac{d E}{T}=0$ cannot define a physical quantity, either.

III. $\oint d Q / T=0$ is a wrong equation based on a wrong calculus deducing So, what is $\oint d Q / T=0$ ?

In textbooks, it has been emphasized that $\oint d Q / T=0$ is a physical result rather than a mathematical conclusion. That is, $\oint d Q / T=0$ cannot be obtained by mathematical derivation. If $\oint d Q / T=0$ is true, this equation should have defined a system state variable. However, the present study has demonstrated the absurdity regarding the definition of a physical quantity by the equation $\oint d Q / T=0$. Thus, the conclusion $\oint d Q / T=0$ doesn't hold.

The key error of entropy is that the mathematical conclusion $\oint d Q / T=0$ cannot be deduced from physics. That is, the conclusion $\oint d Q / T=0$ is untenable to any reversible processes of any working substance engines. The key question is that $\Delta \mathrm{Q} / \mathrm{T} \rightarrow \mathrm{dQ} / \mathrm{T}$ is taken for granted in the process of deducing the relational expression $\oint d Q / T=0$.

It should be noticed from the establishment conditions of differential and the deducing process of entropy that $\Delta \mathrm{Q} / \mathrm{T} \rightarrow \mathrm{dQ} / \mathrm{T}$ is mathematically untenable. The major reasons are as follows:

A. The prerequisite for establishment of differential is the existence of differentiable function. Here $\Delta \mathrm{Q} / \mathrm{T}$ needs to be changed to $\mathrm{dQ} / \mathrm{T}$ on the premise of the existence of a differentiable function $\mathrm{Q}$ $=\mathrm{f}(\mathrm{T})$. However, there is no corresponding differentiable function.

B. Regarding the element calculus, it is an application of calculus, which is established on the 
premise of the existence of differentiable function.

C. Regarding the element calculus, let $\mathrm{Q}$ be a function, which is assumed to be a single-valued function of $T$, i.e., $Q=f(T)$. We get $1 / T d Q=d F(T)$ and $\int_{T} 1 / T d Q=\int_{T} d F(T)$. In this case, $\Delta \mathrm{Q} / \mathrm{T} \rightarrow \mathrm{dQ} / \mathrm{T}$ is tenable.

However, we actually known that for any reversible process, Q is not a single-valued function of $\mathrm{T}$, but follows $Q=f(T, V, P)$. Here, $Q=f(T, V, P)$ is a process quantity which varies with path. It has innumerable forms between the same original and terminal states, and has a unique form for fixed reversible process path. When the given path is fixed, $\mathrm{Q}=\mathrm{f}(\mathrm{T}, \mathrm{V}, \mathrm{P})$ is the system state variable. Hence, regarding $1 / \mathrm{TdQ}=$ $\mathrm{dF}(\mathrm{T}, \mathrm{V}, \mathrm{P}), \mathrm{P}, \mathrm{V}$ and $\mathrm{T}$ are all variables (two variables of $\mathrm{T}, \mathrm{V}$ and $\mathrm{P}$ are generally independent) for any reversible process. Here, only $\int_{\mathrm{T}} \int_{\mathrm{V}} \int_{\mathrm{P}} 1 / \mathrm{TdQ}=\int_{\mathrm{T}} \int_{\mathrm{V}} \int_{\mathrm{P}} \mathrm{dF}(\mathrm{T}, \mathrm{V}, \mathrm{P})$ is meaningful. As in thermodynamics, the P-V diagram should be P-V-T diagram, according which only $\int_{T} \int_{V} \int_{P} 1 / T d Q=\int_{T} \int_{V} \int_{P} d F(T, V, P)$ is clearly meaningful. Then, what does $\int_{\mathrm{T}} 1 / \mathrm{TdQ}=\int_{\mathrm{T}} \mathrm{dF}(\mathrm{T}, \mathrm{V}, \mathrm{P})$ mean? Obviously, it is only meaningful to the calculus of three variables rather than one variable. The P-V-T diagram also shows that it is obviously untenable split or replace any reversible cycles using a series of element reversible Carnot cycles. To any reversible processes, $d Q / T$ (i.e. $d f(T, V, P) / T$ ) is meaningless in itself .

Therefore, the step $\Delta \mathrm{Q} / \mathrm{T} \rightarrow \mathrm{dQ} / \mathrm{T}$ is the misuse of element calculus due to the wrong substitution of P-V-T diagram by $\mathrm{P}-\mathrm{V}$ diagram. The essence of $d \mathrm{Q} / \mathrm{T}$ is $\mathrm{df}(\mathrm{T}, \mathrm{V}, \mathrm{P}) / \mathrm{T}$, but this is meaningless. Without following the principle of calculus, some people by mistake consider the variable $\Delta \mathrm{Q}$, which plays a role only in the reversible isothermal process of Carnot cycle, is identical to the polytropic process function $\mathrm{Q}=$ $\mathrm{f}(\mathrm{T}, \mathrm{V}, \mathrm{P})$ in any reversible process. As a result, the existence of the system status variable, so-called entropy, is taken for granted.

Conclusion: $\oint d Q / T=0$ is neither a mathematical conclusion nor a physical result. The equation $\oint d Q / T=0$ is untenable. Similarly, $\oint d W / T=0$ and $\oint d E / T=0$ are both untenable, and the physical quantity called entropy does not exist.

\section{The Boltzmann entropy}


What is the Boltzmann entropy?

The Boltzmann entropy is used to explain Clausius entropy, with unit $(\mathrm{J} / \mathrm{K})$ of the former taken from the latter. This is because for Boltzmann entropy, the man-made equation $S=k \ln \Omega$ is an artificial combination of a purely digital $\ln \Omega$ and the Boltzmann constant $\mathrm{k}$ with a unit $(\mathrm{J} / \mathrm{K})$. The present study has demonstrated the non-existence of Clausius entropy, which simultaneously denies the Boltzmann entropy.

In statistical physics, the attempt to directly deduce entropy is untenable. On one hand, it involves a key step to translate infinitesimal into differential, which doesn't hold. On the other hand, the unit $(\mathrm{J} / \mathrm{K})$ of entropy (Boltzmann entropy) in statistical physics is transformed from Clausius entropy. So, if Clausius entropy does not exist, there will be no transformation source for the unit $(\mathrm{J} / \mathrm{K})$ of Clausius entropy. As a result, the entropy in statistical physics is only a pure digital, with no physical meaning.

In addition, even if we do not consider the issue regarding the unit, from a pure probability point of view, in the equation $\mathrm{S}=\mathrm{k} \ln \Omega, \Omega$ is the so-called thermodynamic probability, and the calculation of $\Omega$ involves the phase cell division in surpassing space $\mu$. The phase cell is $2 \mathrm{i}$-dimensional and $\mathrm{i}$ the total freedom degree of the molecules within the system. The essence of $\Omega$ calculation is the discretization of the continuous $\mu$ space and the generation of objective meaning. In fact, this approach does not work, and there will be no objective conclusion regardless of the amount of previous work people have done. This is due to the lack of objective, physically meaningful criteria for phase cell division, that is, $\Omega$ has no objective meaning in physics. Together the Liouville theorem and our conclusions indicate that Boltzmann entropy can be taken as a technique for displaying the irreversibility from a purely probabilistic point of view.

\section{The second law of thermodynamics}

The second law of thermodynamics has been expressed by several equivalent statements. Despite these statements proven by numerous facts, we cannot conceal their nature as phenomenological laws. Just as what we express the law of gravity — any object cannot spontaneously move from the lower to higher places, various expressions of the second law are statements of specific phenomena and fail to reveal the unified, essential rules responsible for relevant phenomena. The second law of thermodynamics will be re-stated via a new method. 
1 A. H. Carter, Classical and Statistical Thermodynamics ( Beijing. Qinghua University Press, Beijing, 2007), Chap.1, p. 74-97, Chap.13, p. 235-252.

2 R. K. Su, Statistical Physics ( Beijing. Higher Education Press.,Beijing, 1988 ), Chap. 1, p. 18-42, chap.2, p. $79-85$.

3 Z. C. Wang, Thermodynamics and Statistical Physics (Beijing. Higher Education Press.,Beijing, 2008), Chap. 1, p. 11-14.

4 K. H. Zhao and W.Y. Luo, Thermology (Beijing. Higher Education Press, Beijing, 1998), Chap. 4, p. 178-206.

${ }^{5}$ X. J. Xia, W. R.Chen and S. H. Zhang, Mechanics and Thermology (2nd Vol) (Beijing. Qinghua University Press, Beijing, 1985), Chap. 3-4, p.129-182. 